\title{
IMPORTÂNCIA DO MÉTODO DE OBTENÇÃO DAS DEJEÇÕES DOS TRIATOMÍNEOS NA AVALIAÇÃO DA SUSCETIBILIDADE TRIATOMÍNICA PARA TRYPANOSOMA CRUZI
}

\author{
Ionizete Garcia da Silva, Alejandro Ostermayer Luquetti e \\ Heloisa Helena Garcia da Silva
}

\begin{abstract}
Foi estudada a suscetibilidade de Dipetalogaster maximus, Rhodnius neglectus, $\mathbf{R}$. prolixus, $\mathrm{R}$. robustus, Triatoma infestans $e \mathrm{~T}$. rubrovaria ao Trypanosoma cruzi, através do xenodiagnóstico, em dois pacientes na fase aguda da doença de Chagas. Usaram-se como parâmetros o número de triatomineos infectados e o númera de tripanosomas excretados. A leitura do xenodiagnóstico foi realizada pelos métodos da compressão abdominal e o das dejeções espontâneas. Este foi mais eficiente que aquele, em relação ao número de $\mathrm{T}$. cruzi por lâmina pelo teste de Wilcoxon. Em um dos pacientes, tendo como parâmetro o número de triatomineos infectados, a suscetibilidadedas espécies obedeceu a seguinte ordem: D. maximus $e$ R. neglectus $(100 \%)$, R. robustus $(95 \%)$, R. prolixus $e \mathrm{~T}$. rubrovaria $(90 \%)$ e T. infestans (85\%). Nesse mesmo paciente, a suscetibilidade das espécies de triatomineos, avaliada pelo numero de $\mathrm{T}$. cruzi excretados, usando-se a compressão abdominal, encontra-se na seguinte ordem: R. neglectus, R. prolixus, D. maximus $e$ R. robustus, T. infestans $e$ T. rubrovaria. Pelo método das dejeçzes espontâneas, a ordem foi: $\mathrm{D}$. maximus $e \mathrm{R}$. prolixus, T. rubrovaria, $\mathrm{R}$. robustus, $\mathrm{T}$. infestans $e \mathrm{R}$. neglectus. No outro paciente, esse parâmetro, em ambas as técnicas de leitura, mostrou a mesma ordem de suscetibilidade $(R$. neglectus, $T$. rubrovaria $e \mathrm{~T}$. infestans), porém, o número de tripanosomas excretados foi significativamente maior pelo método das dejeçz̃es espontâneas.
\end{abstract}

Palavras-chaves: Suscetibilidade de triatomineos. Trypanosoma cruzi. Xenodiagnóstico. Doença de Chagas. Fase aguda.

Diante da grande especificidade do xenodiagnóstico na infecção chagásica, tanto no homem quanto em outros mamíferos, vários trabalhos foram realizados para melhorar a sensibilidade deste exame, principalmente com relação à metodologia 7151618 . Outros autores têm estudado a suscetibilidade das espécies de triatomíneos ao Trypanosoma cruzi na busca do triatomíneo mais adequado ao xenodiagnóstico ${ }^{34}$ 691011121314192021 . Neste trabalho, procurou-se verificar o comportamento de Dipetalogaster maximus, Rhodnius neglectus, $R$. prolixus, $R$. robustus, Triatoma infestans e $T$. rubrovaria como meios de cultura (in vivo), utilizados no

\footnotetext{
Departamento de Parasitologia, Instituto de Patologia Tropical e Saúde Pública, Universidade Federal de Goiás, Goiânia, GO.

Endereço para correspondência: Dr. Ionizete Garcia da Silva, Depto. de Parasitologia/IPTSP/UFG, Caixa Postal 131, 74001970 Goiânia, GO.

Recebido para publicação em 20/05/92.
}

xenodiagnóstico, aplicado em dois pacientes (A.S.S. e P.A.B) na fase aguda da doença de Chagas, antes de se iniciar tratamento tripanosomicida específico.

$\mathrm{Na}$ história desses pacientes não havia antecedentes de hospitalização e/ou transfusão sanguínea. Além disso, foi detectada a presença de triatomíneos em suas residências e o sinal de Romaña na paciente A.S.S. Essas evidências levam-nos a crer que esses pacientes adquiriram a doença de Chagas por transmissão natural (via triatomíneo).

\section{MATERIAL E MÉTODOS}

\section{Pacientes}

A.S.S. - era procedente da cidade de Caranhanha, Bahia, do sexo feminino e com 13 anos de idade. Quando chegou ao Hospital das Clínicas (HC) da Universidade Federal de Goiás (UFG), apresentava febre alta e o sinal de Romaña. Pelo exame de sangue (direto) detectou-se a presença de tripanosomas com 
Silva IG, Luquetti AO, Silva HHG. Importância do método de obtenção das dejeç̃es dos triatomíneos na avaliação da suscetibilidade triatomínica para Trypanosoma cruzi. Revista da Sociedade Brasileira de Medicina Tropical 26:19-24, jan-mar, 1993.

cerca de 101 flagelados por lâmina usando-se laminula de $22 \times 22 \mathrm{~mm}$.

P.A.B. - era procedente de Tocantinópolis, Tocantins, do sexo masculino e com 38 anos de idade. Esse paciente foi encaminhado pela Fundação Nacional de Saúde(SUCAM), com exame de sangue positivo para $T$. cruzi. $\mathrm{O}$ exame feito no $\mathrm{HC} / \mathrm{UFG}$ mostrou uma parasitemia de um flagelado para $15 \mu \mathrm{l}$ de sangue.

\section{Origem dos triatomíneos}

As espécies D. maximus, $R$. neglectus, $R$. prolixus, $R$. robustus, $T$. infestans e $T$. rubrovaria foram criadas numa câmara biológica, com temperatura de $30 \pm 1^{\circ} \mathrm{C}$, umidade relativa de $70 \pm 5 \%$ e fotoperíodo de 12 horas. Esses triatomíneos foram criados em frascos plásticos azuis, semitransparentes, resultantes do aproveitamento de garrafas d'água, cilíndricas, medindo $9,0 \mathrm{~cm}$ de diâmetro por $14,5 \mathrm{~cm}$ de altura ${ }^{17}$. $O$ interior desses frascos foi coberto com papel toalha, para absorver o material fecal, e um pedaço de papel rígido dobrado em " $z$ " foi colocado perpendicular à malha que revestia o frasco ${ }^{17}$, para possibilitar o repouso e facilitar a alimentação.

\section{Xenodiagnóstico}

Usou-se para este estudo ninfas de $1^{\circ}$. estádio de $D$. maximus, 10 dias após a eclosão das mesmas, e ninfas de $4^{\circ}$. estádio de $R$. neglectus, $R$. prolixus, $R$. robustus, $T$. infestans e $T$. rubrovaria, criadas nos frascos descritos anteriormente $(9,0 \times 14,5 \mathrm{~cm})$ e alimentadas com sangue de Gallus. gallus domesticus (galinha) ${ }^{17}$. Logo após a ecdise do $4^{\circ}$. estágio, as ninfas eram colocadas em frascos de polietilieno de $3 \times 5 \mathrm{~cm}$, cillíndricos e transparentes, permanecendo nesses por 20 dias, em jejum. Após esse periodo, os frascos eram acondicionados em sacolinhas com alça e essas colocadas na parte flexora do antebraço do paciente, e aí permaneciam cerca de 30 minutos. Colocaram-se 21 triatomíneos de cada espécie, por paciente, e o xenodiagnóstico foi aplicado, diariamente, durante 5 dias na paciente A.S.S., com as espécies $R$. neglectus, $T$. rubrovaria e $T$. infestans, e 7 dias no P.A.B., $\operatorname{com} R$. prolixus, $D$. maximus, $T$. rubrovaria, $R$. robustus, $T$. infestans e $R$. neglectus.

\section{Métodos de leitura do xenodiagnóstico}

Cada triatomíneo foi examinado individualmente, aos 30 e 60 dias, pordois métodos: 1. pelo método da compressão do abdome (M.C.A.), as fezes foram homogeneizadas em solução de cloreto de sódio a $0,6 \%$ e examinadas entre lâmina e lamínula ( $22 \times 22 \mathrm{~mm})$ ao microscópio, com médio aumento $(400 \mathrm{x})$; 2. pelo método das dejeçôes espontâneas (M.D.E.), com auxílio de microplacas plásticas de fundo planoe microscópio de inversão ${ }^{18}$. Esse método consistiu em alimentar os triatomíneos até à repleção total, o que é fundamental para provocar as dejeções (fezes e urina). Para isto, imobilizaram-se os camundongos albinos ${ }^{18}$ e esses foram colocados afixados aos tubos com os triatomíneos. Imediatamente após a alimentação, ou cerca de 3 minutos, os triatomíneos eliminam as fezes, e, cerca de 30 minutos, eliminam a urina. $O$ material, fezes e urina, foi colhido com micropipetador, transferido para microplaca de fundo plano e examinado com médio aumento $(400 \mathrm{x})$ ao microscópio de inversão.

\section{Contagem de tripanosomas}

Através de micropipetador automático, colheuse $5 \mu l$ das dejeções dos triatomíneos e contou-se o número de tripanosomas ${ }^{1} \mathrm{com}$ adaptação ao microscópio do laboratório. Essa adaptação consistiu em contar o número de campos microscópicos, no sentido linear da lamínula, que foi cerca de 49 campos, numa lamínula de $22 \times 22 \mathrm{~mm}$, ocular de 10x e objetiva de 40x. A partir desses dados conheceu-se o diâmetro e o raio $(d / 2=0,2245)$ e $o$ número de campos na lâmina (2.041). Como os campos microscópicos são circulares, há entre eles áreas, às quais convencionou-se chamar de intercampos. A área dos intercampos foi calculada subtraindo-se da área da lamínula $\left(\mathrm{L}=484 \mathrm{~mm}^{2}\right)$ a dos campos $\left(\mathrm{C}=380,13 \mathrm{~mm}^{2}\right)$, que é igual a $103,87 \mathrm{~mm}^{2}$. Transformando-se essa área (intercampos) em campos microscópicos obteve-se 656 campos microscópicos. O número total de campos é igual a 3.057 campos. Estima-se o número de tripanosomas pela contagem em 50 campos, sendo 10 campos em cada ângulo da lamínula e 10 no centro. Para se conhecer o número de tripanosomas nos 3.057 campos, multiplica-se o 
Silva IG, Luquetti AO, Silva HHG. Importância do método de obtenção das dejeçães dos triatomíneos na avaliação da suscetibilidade triatomínica para Trypanosoma cruzi. Revista da Sociedade Brasileira de Medicina Tropical 26:19-24, jan-mar, 1993.

número encontrado de tripanosomas pelo fator 61,15 e tem-se o número de flagelados em $5 \mu 1$.

\section{Análise estatística}

Aplicou-se o teste de Wilcoxon para comparação da eficiência das técnicas de leitura do xenodiagnóstico, e o "U" de Mann-Whitney para comparação da suscetibilidade dos triatomíneos ao T. cruzi.

\section{RESULTADOS}

Os resultados são apresentados na Tabela 1 e Figuras 1 e 2.

A Tabela 1 mostra que a técnica M.D.E. foi mais eficiente do que a M.C.A., em relação ao número de tripanosomas por lâmina (através da leitura), do xenodiagnóstico realizado em ambos pacientes chagásicos na fase aguda, pelo teste de Wilcoxon, com $\mathrm{p}<0,01$.

A carga parasitária dos triatomíneos, determinada através da técnica de leitura M.C.A., em relação ao xenodiagnóstico da paciente na fase aguda $A$.S.S., indicou as espécies que se constituíram como o melhor meio de multiplicação do T. cruzi, e essas encontram-se na ordem decrescente de suscetibilidade: $1^{\circ}$ R. neglectus; $2^{\text {o. }} T$. rubrovaria e $3^{\text {o. }} T$. infestans pelo teste "U"de Mann-Whitney, ao nível de $5 \%$. Observou-se o mesmo quando a leitura do xenodiagnóstico, foi realizada através da técnica M.D.E. (Tabela 1).

Em relação ao paciente P.A.B., os resultados do xenodiagnóstico, através da técnica M.C.A., mostram a suscetibilidade das espécies de triatomíneos ao $T$. cruzi, em ordem decrescente: $1^{\circ}$. $R$. neglectus; $2^{\circ} \cdot R$. prolixus; $3^{\circ}$ D. maximus e $R$. robustus e $4^{\circ}$. $T$. infestans e $T$. rubrovaria, pelo teste de Mann-Whitney, ao nivel de 5\%. Pela técnica M.D.E., as espécies distribuíram-se da seguinte maneira: $1^{\circ} D$. maximus e $R$. prolixus; $2^{\circ}$. T. rubrovaria $; 3^{\circ} R$. robustus; $4^{\circ}$. $T$. infestans e $5^{\circ}$. $R$. neglectus (Tabela 1).

Todos os triatomíneos aplicados na paciente A.S.S. (101 flagelados por lâmina - parasitemia alta) se infectaram, porém, o número de triatomíneos infectados pelo T. cruzi não serviu como parâmetro para caracterizar a suscetibilidade. No entanto, os triatomíneos aplicados no paciente P.A.B. (parasitemia baixa), mostraram que o número de triatomíneos infectados foi diferente entre as espécies, e essas apresentam-se na seguinte ordem: $1^{\text {o. }} D$. maximus e $R$. neglectus $(100 \%) ; 2^{\text {o. }} R$. robustus $(95 \%) ; 3^{\circ}$. $R$. prolixus e $T$. rubrovaria (90\%) e $4^{\circ}$. $T$. infestans (85\%).

As Figuras 1 e 2 evidenciam uma oscilação diária da parasitemia nos pacientes na fase aguda da doença de Chagas, pelo menos das formas infectantes para o triatomíneo, avaliada pelo xenodiagnóstico diário. Na paciente A.S.S. (Tabela 1 e Figura 1),

Tabela 1 - Número médio estimado de Trypanosoma cruzi em $5 \mu$ l de material fecal, através das técnicas de leitura do xenodiagnóstico, M.C.A. (método da compressão abdominal) $e$ M.D.E. (método das dejeções espontâneas), aplicados em chagásicos na fase aguda, usando 21 triatomíneos de cada espécie.

\begin{tabular}{llcc}
\hline \multirow{2}{*}{ Paciente } & Espécie & Número de Trypanosoma cruzi & por lâmina $(5 \mu \mathrm{l})$ \\
\cline { 3 - 4 } & & M.C.A. & M.D.E. \\
\hline A.S.S. & Rhodnius neglectus & 2.900 & 63.000 \\
& Triatoma rubrovaria & 2.100 & 25.000 \\
& Triatoma infestans & 1.400 & 14.000 \\
P.A.B. & Rhodnius prolixus & & \\
& Dipetalogaster maximus & 120 & 31.000 \\
& Triatoma rubrovaria & 45 & 31.000 \\
& Rhodnius robustus & 25 & 10.000 \\
& Triatoma infestans & 45 & 5.900 \\
& Rhodnius neglectus & 29 & 2.600 \\
& & 950 & 350 \\
\hline
\end{tabular}

Teste de Wilcoxon $(\mathrm{p}<0,05)$ 
Silva IG, Luquetti A O. Silva HHG. Importância do método de obtenção das dejeções dos triatomíneos na avaliação da suscetibilidade triatomínica para Trypanosoma cruzi. Revista da Sociedade Brasileira de Medicina Tropical 26:19-24, jan-mar, 1993.

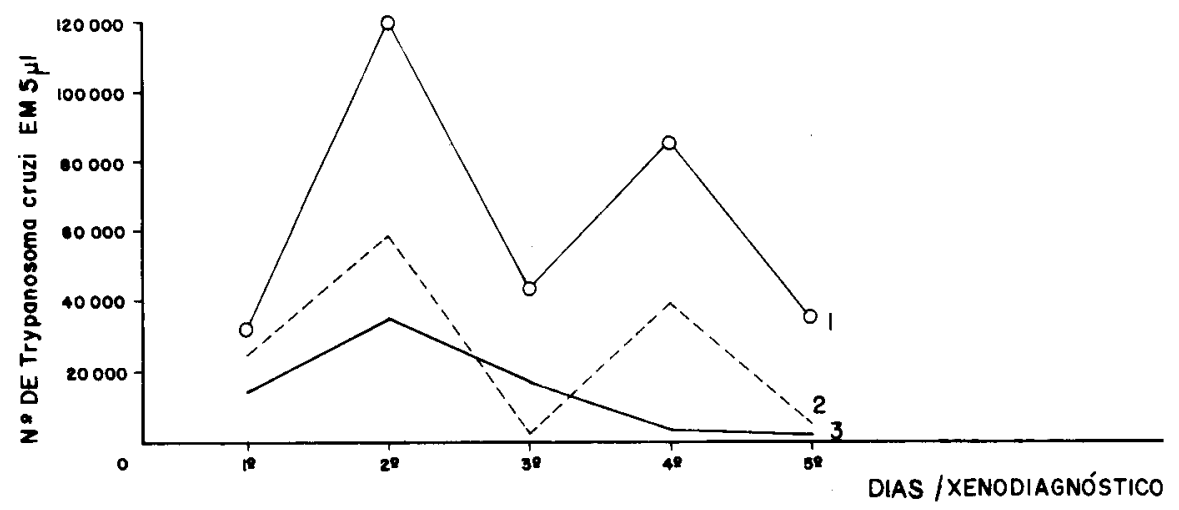

Figura 1 - Número estimado de Trypanosoma cruzi, em ninfas de Rhodnius neglectus (1), Triatoma rubrovaria (2) e Triatoma infestans (3), através do xenodiagnóstico diário no chagásico na fase aguda (A.S.S.).

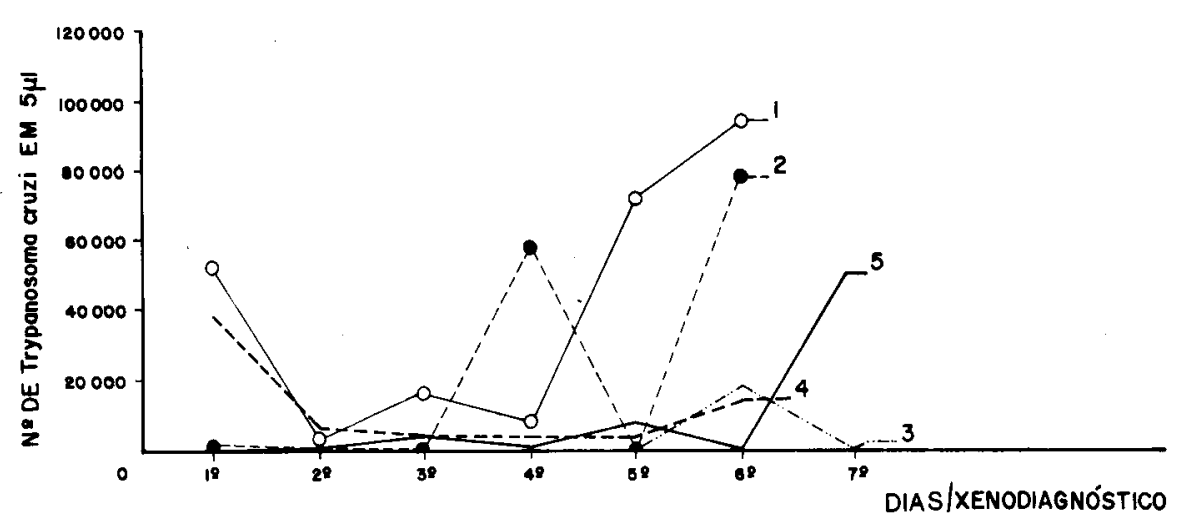

Figura 2 - Número médio estimado de Trypanosoma cruzi, em ninfas de Dipetalogaster maximus (1), Rhodinius prolixus (2), Rhodnius robustus (3), Triatoma infestans (4) $e$ Triatoma rubrovaria (5), através doxenodiagnóstico diário no chagásico na fase aguda (P.A.B.).

houve um pico da parasitemia nos $2^{\text {o. }}$ e $4^{\text {o. }}$ xenodiagnósticos, correspondentes aos $2^{\circ}$ e $4^{\circ}$. dias entre a internação e o início do tratamento específico. Na Figura 1, fica nítido também que $R$. neglectus comportou-se como o melhor meio de cultura (in vivo) para replicar o T. cruzi. No paciente P.A.B. (Tabela 1), os dados obtidos através do método de leitura M.D.E. evidenciaram que as espécies $D$. maximus e $R$. prolixus comportaram-se como os melhores meios replicadores de T. cruzi.

\section{DISCUSSÃO}

A suscetibilidade de triatomíneos ao $T$. cruzi tem sido estudada por diversos pesquisadores 25614 e esses mostraram que as espécies de triatomíneos mais suscetíveis à cepa "Y" de $T$. cruzi eram da mesma região. Outros ${ }^{21}$ obtiveram resultados inversos aos anteriores, e similares aos desse trabalho com D. maximus e R. prolixus. As espécies silvestres de triatomíneos têm-se mostrado mais suscetíveis 
Silva IG, Luquetti AO, Silva HHG. Importância do método de obtenção das dejeções dos triatomíneos na avaliação da suscetibilidade triatomínica para Trypanosoma cruzi. Revista da Sociedade Brasileira de Medicina Tropical 26:19-24, jan-mar, 1993.

ao $T$. cruzi do que as domiciliadas ${ }^{12}$. Esses resultados são concordantes com os deste trabalho, no entanto, existe investigação demonstrando o contrário 9 comportamento de $D$. maximus, como uma das espécies mais suscetíveis ao $T$. $c r u z i^{3}{ }^{48}$, é concordante com os resultados obtidos neste trabalho. Desta forma, a suscetibilidade deve estar associada a vários fatores que devem ser investigados simultaneamente e que, até o momento, não estão bem esclarecidos. Ressalta-se que a técnica de leitura do xenodiagnóstico tem papel importante no estudo da suscetibilidade das espécies de triatomíneos ao $T$. cruzi, quando se usa como parâmetro o número de tripanosomas excretados. Neste trabalho, a técnica de leitura M.D.E. mostrou-se mais eficiente do queo M.C.A. e, no paciente P.A.B., determinou outra ordem de suscetibilidade. O M.D.E. apresenta as seguintes vantagens sobre o M.C.A.:

1. não utiliza solução salina para diluição do material fecal;

2. possibilidade de se examinar separadamente as fezes e urina, o que não é possível realizar com o M.D.E.;

3. a urina apresenta predominância de formas tripomastigotas e, praticamente, livre de contaminação por fungos e bactérias, o que é fundamental na obtenção de inóculos e infecção de animais, possibilitando outras investigações;

4. pode ser utilizada para isolamento e/ou clonagem de cepas;

5. obtém-se maior quantidade de flagelados (Tabela 1);

6. permite examinar o triatomíneo sem provocar traumatismo ou morte do inseto, o que é de fundamental importância para se estudar a interação parasita-hospedeiro e a determinação de curva parasitêmica do vetor infectado, durante os estádios ninfais e sua longevidade.

$D$. maximus, $R$. neglectus e $R$. prolixus foram as espécies que se comportaram como os melhores meios de cultura (in vivo) para replicação do $T$. cruzi. Destas três, sugere-se o uso de $D$. maximus, pelas seguintes razões: 1. por provocar menor reação cutânea (picada); 2. por não haver possibilidade de se contaminar na criação, uma vez que se utilizam ninfas de $1^{\text {o. }}$ estádio, provenientes de ovos isolados em incubadoras; 3 . gasta menor tempo para atingir a repleção total, cerca de 10 minutos.

\section{SUMMARY}

Two patients on the acute phase of Chagas' disease were submitted toxenodiagnosis examination with different species of triatomine bugs, in order to study bug susceptibility to Trypanosoma cruzi. Species used were Dipetalogaster maximus, Rhodnius neglectus, $\mathbf{R}$. prolixus, $R$. robustus, Triatoma infestans and T. rubrovaria. Both the number of infected bugs, and the number of excreted trypanosomes by bug, were used as parameters for evaluation of species susceptibility. Xenodiagnosis reading was performed by two methods, the classic abdominal compression and the spontaneous dejection method; this was more efficient than the former in relation to the number of parasites per wet smear (by Wilcoxon test). When susceptibility was evaluated by the number of infected bugs, in one of the patients all $\mathrm{D}$. maximus and $R$. neglectus became infected (100\%), 95\% of R. robustus, $90 \%$ of $\mathrm{R}$. prolixus and $\mathrm{T}$. rubrovaria, and $85 \%$ of $\mathrm{T}$. infestans. When evaluation was performed through the number of excreted parasites in this patient with reading by abdominal compression, the higher susceptibility was with R. neglectus, followed by R. prolixus, D. maximus and $\mathrm{R}$. robustus, $\mathrm{T}$. infestans and $\mathrm{T}$. rubrovaria; reading by spontaneous dejection yielded better results for $\mathrm{D}$. maximus and $\mathrm{R}$. prolixus followed by $\mathrm{T}$. rubrovaria, $\mathrm{R}$. robustus, $\mathrm{T}$. infestans and $\mathrm{R}$. neglectus. For the other patient susceptibility evaluated by the number of excreted parasites was similar by both reading methods and did show the same susceptibility pattern $(\mathbf{R}$. neglectus and then $\mathrm{T}$. rubrovaria and $\mathrm{T}$. infestans), but the number of excreted trypanosomes was much higher by the spontaneous dejection method.

Key-words: Susceptibility of triatomines. Trypanosoma cruzi. Xenodiagnosis. Chagas' disease. Acute phase.

\section{AGRADECIMENTOS}

Os autores agradecem ao Dr. Anis Rassi pelo encaminhamento dos pacientes.

\section{REFERÊNCIAS BIBLIOGRÁFICAS}

1. Brener $Z$. Contribuição ao estudo da terapêutica experimental da doença de Chagas. Tese de Docência-Livre. Faculdade de Odontologia e Farmácia de Minas Gerais, Belo Horizonte, 1961.

2. Cerisola JA, Rhowedder RW, Prado CE. Rendimento del xenodiagnóstico en la infección crónica humana utilizando ninfas de diferentes espécies de triatomíneos. Boletin Chileno de Parasitologia 26:5758,1971 . 
Silva IG, Luquetti AO, Silva HHG. Importância do método de obtenção das dejeções dos triatomíneos na avaliação da suscetibilidade triatomínica para Trypanosoma cruzi. Revista da Sociedade Brasileira de Medicina Tropical 26:19-24, jan-mar, 1993.

3. Cuba CAC, Alvarenga NJ, Barreto AC, Marsden PD, Chiarini C. Nuevos estudios comparativos entre Dipetalogaster maximus e Triatoma infestans en xenodiagnostico de la infección chagasica crónica humana. Revista do Instituto de Medicina Tropical de São Paulo 20:145-151, 1978.

4. Cuba CAC, Alvarenga NJ, Barreto AC, Marsden PD, Gama MP. Dipetalogaster maximus (Hemiptera, Triatominae) for xenodiagnosis of patients with serologically detectable Trypanosoma cruzi infection. Transactions of the Royal Society of Tropical Medicine and Hygiene 73:524-527, 1979.

5. Dias E. Xenodiagnósticos seriados em cães infectados com amostras venezuelanas de "Schizotrypanum cruzi". Brasil Médico 54:859-861, 1940.

6. Little JW, Tay J, Biaafi F. A study on the susceptibility of triatominae bugs to some Mexican strains of Trypanosoma cruzi. Journal of Medical Entomology 3:252-255, 1966.

7. Maekelt GA. A modified procedure of xenodiagnosis for Chagas' disease. The American Journal of Tropical Medicine and Hygiene 13:11-15, 1964.

8. Marsden PD, Barreto AC, Cuba CC, Gama MB, Akers J. Improvements in routine xenodiagnosis with first isntar Dipetalogaster maximus (Uhler, 1894)(Triatominae). The American Journal of Tropical Medicine and Hygiene 28:649-652, 1979.

9. Mello DA, Chiarini C. Suscetibilidade dos diferentes estádios evolutivos de Triatoma sordida (Stal, 1859) e de Rhodnius neglectus (Lent, 1954), à infecção pelo Trypanosoma cruzi. Revista Brasileira de Biologia 40:327-334, 1980.

10. Miles MA, Patterson JW, Marsden PD, Minter MD. A comparison of Rhodnius prolixus, Triatoma infestans and Panstrongylus megistus in the xenodiagnosis of a chronic Trypanosoma (Schizotrypanum) cruzi infection in a Rhesus monkey (macaca mulata). Transactions of the Royal Society of Tropical Medicine and Hygiene 69:377382, 1975.

11. Neal RA, Miles RA. The numbers of trypomastigotes of Trypanosoma cruzi required to infect Rhodnius prolixus. Revista do Instituto de Medicina Tropical de São Paulo 19:177-181, 1977.
12. Perlowagora-Szumlewicz A, Muller CA. Studies in search a suitable experimental insect model for xenodiagnosis of hosts with nine triatominae species of animals with acute infections by Trypanosoma cruzi. Memórias do Instituto Oswaldo Cruz 77:37$53,1982$.

13. Phillips NR, Bertran DS. Laboratory studies of Trypanosoma cruzi infections in: Rhodnius prolixus - larvae and adults; in: Triatoma infestans, T. protracta and $T$. maculata - adults. Journal of Medical Entomology 4:168-174, 1967.

14. Ryckman RE. Epizootiology of Trypanosoma cruzi in Southwestern North America. V. Host parasite specificity between Trypanosoma and Triatominae (Kinetoplastida:Tripanosomatidae) (Hemiptera:triatominae). Journal of Medical Entomology 2:96-99, 1965.

15. Schenone H, Alfaro E, Reyes H, Taucher E. Valor del xenodiagnóstico en la infección chagásica crónica. Boletín Chileno de Parasitologia 32:63-66, 1977.

16. Schenone H, Rojo M, Rojas A, Concha L. Positividad diurna y nocturna del xenodiagnóstico en un paciente con infección chagásica crónica de parasitemia permanente. Boletín Chileno de Parasitologia 32:6366, 1977.

17. Silva IG. Influência da temperatura na biologia de triatomíneos. I. Triatoma rubrovaria (Blanchard,1843)(Hemiptera, Reduviidae). Revista Goiana de Medicina 31:1-37, 1985.

18. Silva IG. Nova técnica para leitura de xenodiagnóstico. Revista Goiana de Medicina 36:3539, 1990.

19. Silva IG, Ferrreira IR. Influência da fonte sanguínea na multiplicação da cepa "Y"de Trypanosoma cruzi em Triatoma infestans (Klug,1834) e Rhodnius neglectus (Lent,1954). Revista Goiana de Medicina 36:40-48, 1990.

20. Torres $M$. Alguns fatos que interessam à epidemiologia da moléstia de Chagas. Memórias do Instituto Oswaldo Cruz 7:120-136, 1915.

21. Zeledón R, Vieto PL. Suscetibilidad de várias especies de triatomíneos a una cepa costarricence de Schizotrypanum cruzi. Revista de Biologia Tropical 5:195-199, 1957. 